\title{
ARTICLE
}

Epidemiology

\section{Adiposity and risks of colorectal and small intestine cancer in Chinese adults: a prospective study of 0.5 million people}

\author{
Yuanjie Pang ${ }^{1}$, Christiana Kartsonaki ${ }^{1,2}$, Yu Guo ${ }^{3}$, Yiping Chen ${ }^{1,2}$, Ling Yang ${ }^{1,2}$, Zheng Bian ${ }^{3}$, Fiona Bragg ${ }^{1}$, lona Y. Millwood ${ }^{1,2}$, \\ Enke $\mathrm{Mao}^{4}$, Yilei $\mathrm{Li}^{5}$, Liya Shi ${ }^{6}$, Junshi Chen ${ }^{7}$, Liming $\mathrm{Li}^{3,8}$, Michael V. Holmes ${ }^{1,2,9}$ and Zhengming Chen
}

\begin{abstract}
BACKGROUND: Uncertainty remains about the associations of adiposity with intestinal cancer in China and by its anatomical subtype.

METHODS: The prospective China Kadoorie Biobank recorded 3024 incident cases of colorectal (CRC) and 143 cases of small intestine (SIC) cancer during a 10-year follow-up among 509568 participants without prior cancer at baseline. Cox regression was used to estimate adjusted hazard ratios (HRs) for specific cancers associated with adiposity.

RESULTS: Overall mean body mass index (BMI) was $23.7 \mathrm{~kg} / \mathrm{m}^{2}$. BMI was positively associated with CRC (HR per SD 1.10 [95\% Cl 1.06-1.14]), colon (1.13 [1.07-1.18]), and rectal (1.07 [1.02-1.13]) cancer. For waist circumference, the corresponding HRs per SD were 1.14 (1.10-1.18), $1.18(1.13-1.24)$, and 1.11 (1.05-1.16), respectively. The adjusted HRs were somewhat greater in men than women. Adiposity was positively, but non-significantly, associated with SIC risk.

CONCLUSIONS: Among relatively lean Chinese adults, adiposity was associated with risks of colon and rectal cancer, with the associations somewhat stronger in men than women.
\end{abstract}

British Journal of Cancer (2018) 119:248-250; https://doi.org/10.1038/s41416-018-0124-8

\section{INTRODUCTION}

High body fatness has been established as a cause of colorectal cancer (CRC). ${ }^{1}$ Uncertainty remains, however, as to whether the association differs by sex or by its anatomical site. Moreover, the evidence is rather limited in the East Asian population, ${ }^{2}$ particularly China, where the mean levels of adiposity in the adult population are still much lower than those in the Western population. Despite the low incidence, small intestine cancer (SIC) may arise from the same origin as that of $C R C$, and therefore may share similar risk factors. ${ }^{3}$ Nonetheless, prospective evidence on the relevance of adiposity for SIC risk has been inconclusive. ${ }^{4}$ The present study examined the associations of general and central adiposity with risks of CRC and SIC within the China Kadoorie Biobank (CKB) population of 0.5 million adults, both overall and by sex and anatomical site.

\section{MATERIALS AND METHODS}

\section{Study population and follow-up}

Overall, 512891 men and women, aged 30-79 years, were recruited to the CKB from 10 areas in China during 2004-2008. ${ }^{5}$ Extensive data were collected on all participants at baseline through interviewer-administered laptop-based questionnaires, covering socioeconomic and demographic factors, lifestyle habits, personal and family medical history, and current medication. Physical measurements (including anthropometric measures, lung function, heart rate, and blood pressure) and collection of a nonfasting blood sample were undertaken for each participant.

Long-term follow-up data on cause-specific mortality and major morbidity, as well as any episodes of hospitalisation, were collected through linkages via unique national ID number with registries and health insurance databases. The present study excluded participants with a prior history of cancer $(n=2577)$ or missing/implausible adiposity values $(n=746)$, leaving 509568 participants for the main analysis.

\section{Exposures and outcomes}

Adiposity measures included general (e.g. body mass index [BMI], percent body fat, height adjusted weight) and central adiposity (e.g. waist circumference [WC], waist-to-hip ratio [WHR]). Cancer outcomes included incident cases of CRC $(C 18-20, n=3024)$, colon cancer $(C 18, n=1745)$, rectal cancer $(C 20, n=1716)$, and SIC $(C 17, n=143)$ (Supplementary Table S1). Cox regression was used to estimate adjusted hazard ratios (HRs) for specific cancers associated with adiposity, stratified by age-at-risk, sex, and region, and adjusted for age at baseline, education, smoking, alcohol,

\footnotetext{
${ }^{1}$ Clinical Trial Service Unit \& Epidemiological Studies Unit (CTSU), Nuffield Department of Population Health, University of Oxford, Oxford, UK; ${ }^{2}$ Medical Research Council

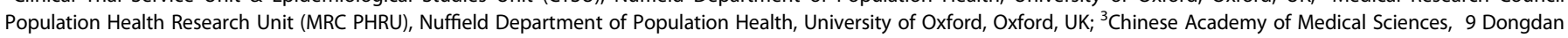

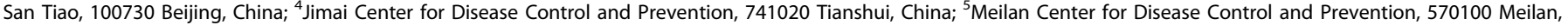

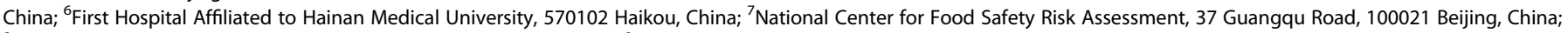

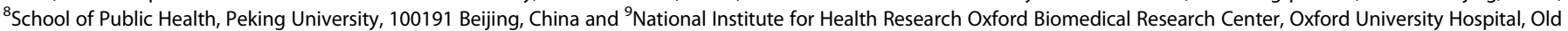
Road, OX3 7LE Oxford, UK

Correspondence: Christiana Kartsonaki (christiana.kartsonaki@ndph.ox.ac.uk)
} 


\begin{tabular}{|c|c|c|c|c|c|c|c|c|c|c|}
\hline & \multicolumn{3}{|l|}{ Men } & \multicolumn{3}{|l|}{ Women } & \multicolumn{2}{|l|}{ Men } & \multicolumn{2}{|c|}{ Women } \\
\hline & Median $^{a}$ & No. $^{b}$ & $\mathrm{HR}(95 \% \mathrm{CI})^{\mathrm{c}}$ & Median & No. & $\mathrm{HR}(95 \% \mathrm{Cl})$ & No. & $\mathrm{HR}(95 \% \mathrm{Cl})$ & No. & $\mathrm{HR}(95 \% \mathrm{Cl})$ \\
\hline \multicolumn{11}{|l|}{ BMI } \\
\hline$<20.0$ & 19.0 & 100 & $1.03(0.84,1.26)$ & 18.9 & 90 & $0.88(0.71,1.08)$ & 124 & $1.08(0.90,1.31)$ & 87 & $0.87(0.70,1.07)$ \\
\hline 22.5 to $<25.0$ & 23.7 & 253 & $1.31(1.16,1.48)$ & 23.7 & 233 & $0.94(0.83,1.07)$ & 237 & $1.10(0.97,1.25)$ & 230 & $0.99(0.87,1.13)$ \\
\hline 25.0 to $<27.5$ & 26.0 & 188 & $1.32(1.14,1.53)$ & 26.0 & 195 & $1.05(0.91,1.21)$ & 191 & $1.23(1.06,1.42)$ & 168 & $0.97(0.83,1.12)$ \\
\hline$\geq 27.5$ & 28.8 & 136 & $1.69(1.41,2.01)$ & 29.1 & 179 & $1.23(1.05,1.43)$ & 134 & $1.49(1.24,1.77)$ & 156 & $1.12(0.95,1.32)$ \\
\hline 1-SD increment ${ }^{d}$ & & & $1.18(1.10,1.26)$ & & & $1.10(1.03,1.17)$ & & $1.11(1.03,1.19)$ & & $1.05(0.98,1.12)$ \\
\hline \multicolumn{11}{|l|}{$w c^{e}$} \\
\hline Quintile 5 & 95.0 & 249 & $1.86(1.62,2.12)$ & 91.5 & 243 & $1.23(1.08,1.41)$ & 245 & $1.52(1.32,1.74)$ & 230 & $1.23(1.07,1.41)$ \\
\hline 1-SD increment ${ }^{d}$ & & & $1.28(1.19,1.37)$ & & & $1.12(1.04,1.19)$ & & $1.18(1.10,1.27)$ & & $1.04(0.97,1.12)$ \\
\hline \multicolumn{11}{|l|}{$W H R^{f}$} \\
\hline Quintile 1 & 0.82 & 104 & $0.75(0.61,0.91)$ & 0.78 & 155 & $1.22(1.03,1.43)$ & 126 & $0.91(0.76,1.09)$ & 107 & $0.78(0.64,0.94)$ \\
\hline Quintile 2 & 0.87 & 147 & $1.00(0.85,1.18)$ & 0.83 & 134 & $1.00(0.84,1.19)$ & 149 & $1.00(0.85,1.17)$ & 150 & $1.00(0.85,1.18)$ \\
\hline Quintile 3 & 0.90 & 141 & $1.05(0.89,1.24)$ & 0.86 & 131 & $1.10(0.93,1.30)$ & 174 & $1.30(1.12,1.51)$ & 129 & $0.95(0.80,1.12)$ \\
\hline Quintile 4 & 0.93 & 214 & $1.28(1.12,1.46)$ & 0.89 & 192 & $1.24(1.08,1.43)$ & 197 & $1.21(1.05,1.39)$ & 202 & $1.11(0.96,1.27)$ \\
\hline Quintile 5 & 0.98 & 244 & $1.38(1.21,1.57)$ & 0.95 & 283 & $1.39(1.22,1.57)$ & 241 & $1.41(1.24,1.61)$ & 241 & $0.96(0.84,1.10)$ \\
\hline 1 -SD increment ${ }^{d}$ & & & $1.20(1.14,1.27)$ & & & $1.07(1.00,1.14)$ & & $1.15(1.09,1.23)$ & & $1.05(0.98,1.12)$ \\
\hline
\end{tabular}

physical activity, and dietary factors (fresh fruits, vegetables, red meat, and dairy products). A more detailed description of the methods and data source is presented in Supplementary Material.

\section{RESULTS}

Overall mean (SD) BMI and WC were 23.7 (3.3) $\mathrm{kg} / \mathrm{m}^{2}$ and $80.3(9.8) \mathrm{cm}$, respectively. Participants with higher BMI were more likely to have higher systolic blood pressure and random plasma glucose (RPG), and to have prevalent diabetes and a history of cardiovascular disease or hypertension (Supplementary Table S2). The standardised incidence rates of CRC were higher in men and in urban areas (Supplementary Table S3).

There were positive associations of adiposity, irrespective of how it was measured, with the risk of CRC (Supplementary Table S4). For both colon and rectal cancer, the associations of BMI appeared stronger in men than in women (Table 1), although the differences were non-significant (Supplementary Table S5, $p$ for heterogeneity $=0.13$ and 0.27 ). Similarly, the positive associations of central adiposity (WC and WHR) were stronger in men than in women (Table 1 and Supplementary Table S5, $p$ for heterogeneity $=0.007-0.04)$. In both sexes, the associations of adiposity with colon cancer appeared stronger than with rectal cancer (Table 1), albeit non-significant (Supplementary Table S5, $p$ for heterogeneity $=0.12-0.69)$. When both BMI and WC were included in the same model (Supplementary Table S6), the risk estimates for WC changed little, while the estimates for BMI attenuated and became non-significant.

Similarly, hip circumference $(\mathrm{HC})$, percent body fat, height adjusted weight, height, weight-to-height ratio, and weight change, since age 25, all showed positive associations with the risk of CRC, but the association was weaker for BMI at age 25 than for other adiposity measures (Supplementary Table S7). When further adjusting for BMI, the positive associations persisted for WC, HC, and WHR, but not for other measures (Supplementary Figure S1). For these measures of adiposity, the associations were similar for colon and rectal cancer (Supplementary Figure S1), and for proximal and distal colon cancer (Supplementary Table S8).

BMI showed a non-significant, positive trend with the risk of SIC (HR per SD 1.06 [0.89-1.25], Supplementary Table S4). Similarly, there were positive trends for other adiposity traits (Supplementary Table S4 and S7), and the associations were strongest for WC and HC (HR per SD 1.11 [0.94-1.32] and 1.21 [1.01-1.45], respectively).

The associations of adiposity with risks of CRC and SIC changed little when additionally adjusting for diabetes or RPG (Supplementary Table S9), and were also similar in urban and rural areas (Supplementary Table S10).

\section{DISCUSSION}

In this relatively lean Chinese population, we found that general and central adiposity were positively associated with the risk of 
CRC, with somewhat stronger associations in men and for colon cancer. General and central adiposity showed trends towards higher risk of SIC, in agreement with previous studies in Western population and in Asia (Supplementary Table S11).

The sex- and site-specific findings in the present study are generally consistent with those in previous studies (Supplementary Figure S2). A recent meta-analysis of 38 prospective cohort studies (> 71,000 cases) reported a $6 \%$ higher risk of CRC per $5 \mathrm{~kg} /$ $\mathrm{m}^{2}$ higher $\mathrm{BMI}^{2}$ with somewhat stronger associations in men than women and for colon than rectal cancer. ${ }^{2}$ Despite the much lower mean BMI in China than in North America or Europe, ${ }^{6}$ our risk estimates for $\mathrm{BMI}$ appeared somewhat stronger than those in the Western population (per $5 \mathrm{~kg} / \mathrm{m}^{2}: 1.14$ [1.09-1.21] in CKB vs 1.04 [1.02-1.06] in Europe and 1.05 [1.03-1.07] in North America), but was broadly consistent with those in Asian studies included in that meta-analysis (1.09 [1.01-1.18]). ${ }^{2}$

For WC and WHR, the risk estimates reported in the metaanalysis were somewhat weaker than our estimates. ${ }^{2}$ Moreover, unlike in the present study, they differed little by sex and by anatomical site. ${ }^{2}$ Of all included studies, four studies showed that WC or WHR was more important than BMI in predicting the risk of CRC, while the converse was true for the other two studies. ${ }^{2}$ Our study showed that the association of BMI with CRC risk attenuated when further adjusting for WC, while the association of WC persisted when further controlling for BMI.

The strengths of the CKB include a prospective design, a large and diverse study population, a large number of CRC cases by subtypes, ability to assess a range of adiposity measures, and careful adjustment for other risk factors. Although BMI may not be a good proxy for body fat, ${ }^{7,8}$ we found it is as good as other measures of adiposity, including percent body fat in predicting the risk of CRC. Moreover, we were also able to adjudicate $\sim 30 \%$ of all CRC and SIC cancer cases (94\% adenocarcinoma, Supplementary Table S12), and showed similar associations for total and adjudicated outcomes (Supplementary Table S13). However, our results do not necessarily indicate causality and residual confounding may still exist.

In conclusion, we showed that both general and central adiposity were associated with the risk of CRC in this relatively lean adult Chinese population. Our study suggested that adiposity, particularly central adiposity, might be associated with higher risk of SIC.

\section{ACKNOWLEDGEMENTS}

The chief acknowledgement is to the participants, the project staff, and the China National Centre for Disease Control and Prevention (CDC) and its regional offices for access to death and disease registries. The Chinese National Health Insurance scheme provided electronic linkage to all hospital admission data.

\section{AUTHOR CONTRIBUTIONS}

C.K., L.L., and Z.C. had full access to the data. Y.P., C.K., M.H., and Z.C. conducted data analysis, and are responsible for accuracy of the results and the decision to submit for publication. All authors were involved in study design, conduct, long-term follow-up, review and coding of disease events, interpretation of the results, or writing the report.

\section{ADDITIONAL INFORMATION}

Supplementary information is available for this paper at https://doi.org/10.1038/ s41416-018-0124-8.

Competing interests: The authors declare no competing interests.
Availability of data and materials: The materials are available upon request; some restrictions will apply.

Ethics approval and consent to participate: Central ethical approvals were obtained from Oxford University and the China National CDC. Approvals were also obtained from institutional research boards at the local CDCs in ten areas: Qingdao, Qingdao CDC; Heilongjiang, Provincial CDC; Hainan, Provincial CDC; Jiangsu, Provincial CDC; Guangxi, Provincial CDC; Sichuan, Provincial CDC; Gansu, Provincial CDC; Henan, Provincial CDC; Zhejiang, Provincial CDC; and Hunan, Provincial CDC.

Publisher's note: Springer Nature remains neutral with regard to jurisdictional claims in published maps and institutional affiliations.

Funding: This study is funded by Baseline survey Kadoorie Charitable Foundation, Hong Kong. Long-term continuation is by UK Wellcome Trust (088158/Z/09/Z, 104085/Z/14/Z), Chinese Ministry of Science and Technology (2011BAI09B01, 201214), and Chinese National Natural Science Foundation (81390541). The British Heart Foundation, UK Medical Research Council, and Cancer Research UK provided core funding to the Oxford CTSU. Fiona Bragg acknowledges support from the BHF Centre of Research Excellence, Oxford. Michael Holmes is supported by a British Heart Foundation Intermediate Clinical Research Fellowship (FS/18/23/ 33512) and the National Institute for Health Research Oxford Biomedical Research Centre.

\section{REFERENCES}

1. World Cancer Research Fund International/American Institute for Cancer Research. Continuous Update Project Report: Diet, Nutrition, Physical Activity and Colorectal Cancer 2017. Available at: wcrf.org/colorectal-cancer-2017. All CUP reports are available at wcrf.org/cupreports.

2. Abar, L., Vieira, A.R., Aune, D., Sobiecki, J.G., Vingeliene, S., Polemiti, E., et al. Height and body fatness and colorectal cancer risk: an update of the WCRF-AICR systematic review of published prospective studies. Eur. J. Nutr. (2017). https://doi. org/10.1007/s00394-017-1557-1

3. Neugut, A. I., Jacobson, J. S., Suh, S., Mukherjee, R. \& Arber, N. The epidemiology of cancer of the small bowel. Cancer Epidemiol. Biomarkers Prev. 7, 243-251 (1998).

4. Boffetta, P., Hazelton, W.D., Chen, Y., Sinha, R., Inoue, M., Gao, Y.T., et al. Body mass, tobacco smoking, alcohol drinking and risk of cancer of the small intestine-a pooled analysis of over 500,000 subjects in the Asia Cohort Consortium. Ann. Oncol. 23, 1894-98 (2011).

5. Chen, Z., Chen, J., Collins, R., Guo, Y., Peto, R., Wu, F., et al. China Kadoorie Biobank of 0.5 million people: survey methods, baseline characteristics and long-term follow-up. Int J. Epidemiol. 40, 1652-66 (2011).

6. Ng, M., Fleming, T., Robinson, M., Thomson, B., Graetz, N., Margono, C., et al. Global, regional, and national prevalence of overweight and obesity in children and adults during 1980-2013: a systematic analysis for the Global Burden of Disease Study 2013. Lancet 384, 766-81 (2014).

7. Frankenfield, D. C., Rowe, W. A., Cooney, R. N., Smith, J. S. \& Becker, D. Limits of body mass index to detect obesity and predict body composition. Nutrition 17, 26-30 (2001).

8. Deurenberg, P., Deurenberg-Yap, M. \& Guricci, S. Asians are different from Caucasians and from each other in their body mass index/body fat per cent relationship. Obes. Rev. 3, 141-146 (2002).

Open Access This article is licensed under a Creative Commons Attribution 4.0 International License, which permits use, sharing, adaptation, distribution and reproduction in any medium or format, as long as you give appropriate credit to the original author(s) and the source, provide a link to the Creative Commons license, and indicate if changes were made. The images or other third party material in this article are included in the article's Creative Commons license, unless indicated otherwise in a credit line to the material. If material is not included in the article's Creative Commons license and your intended use is not permitted by statutory regulation or exceeds the permitted use, you will need to obtain permission directly from the copyright holder. To view a copy of this license, visit http://creativecommons. org/licenses/by/4.0/.

(c) The Author(s) 2018 